\title{
Biosynthesis of Copper Nanoparticles by using Aloe Barbadensis Leaf Extracts
}

\author{
Madiha Batool*, Zahid Qureshi, Farwa Hashmi and Nida Mehboob \\ Department of Chemistry, Government College University, Pakistan
}

Received: 笽April 06, 2018; Published: 眥April 19, 2018

*Corresponding author: Madiha Batool, Department Of Chemistry, Government College University, Lahore, Pakistan

\begin{abstract}
Development of green nanotechnology is generating interest of researchers toward eco-friendly biosynthesis of nanoparticles. In this study, biosynthesis of stable copper nanoparticles was done using aloe barbadensis leaf extract. First, we prepared leaf extract of aloe barbadensis in deionized water. This extract added to $1 \mathrm{mMol}$ of copper sulfate solution and we observed the change in color of the solution from colorless to colored solution. The present study tracing of an object is a green synthesis of copper nanoparticles by the interaction of leaf extract and copper salt. Biosynthesis of nanoparticle put forward a cost-free and environmentally suitable method of nanoparticle synthesis. Powder X-ray diffraction and SEM analysis showed that synthesized CuO nanoparticles with an average particle size of $60 \mathrm{~nm}$. Shape of copper nanoparticles was spherical and cubic and their range was $80-120 \mathrm{~nm}$. EDX of synthesized nanoparticles showed copper $38 \%$. UV spectrophotometer confirms peak of the copper nanoparticle.
\end{abstract}

Abbreviations: Aloe Barbadensis; SEM; Copper oxide nanoparticles; Green synthesis; XRD

\section{Introduction}

Recent advances in the field of science and technology, particularly nanotechnology, have to lead to the development a new concept of synthesizing nanosized particles of desired size and shape [1]. Hence, there is scope to develop new methods for the synthesis of nanoparticles which should be required inexpensive reagent, less drastic reaction condition and eco-friendly. In recent years, $\mathrm{Cu}$ nanoparticles have attracted much attention of researchers due to its application in wound dressings and biocidal properties. Nanotechnology deals with the manipulation of matter at low size normally less than $100 \mathrm{~nm}$. Metallic nanoparticles can be prepared by the chemical and physical method. These methods have certain flaws like toxic chemicals and also dangerous to the environment. Developing research in green chemistry employed prominent part in nanotechnology to attain benefit to society. Surface area and mass ratios increase adsorption property. Therefore, the need for the development of a clean, reliable, biocompatible, benign and eco-friendly process to synthesize nanoparticles forced many researchers to develop green chemistry and bioprocesses [2].

Green synthesis has been engaged in synthesis of highly stabilized nanoparticles. Nanoparticles integrity maintenance is the challenge of green synthesis. Copper nanoparticles were synthesized by leaf extract of aloe vera plant. The green synthesis of the copper nanoparticle is a speedy, economically feasible, and efficient method. Phenolic content in plant extracts dissolved in water, degradable and catalyzed synthesis of the nanoparticle as capping and a reducing agent [3].

Synthesis of metal Nanoparticles has been an area of interest in recent past as Nanoparticles show unusual structural, electrical, optical and magnetic properties. The unique properties of Nanoparticles can be tailored to the growth of Nanoparticles. So it is the need of the hour to develop certain green methods of synthesis which have lesser detrimental effects on the environment [4] (Figure 1).

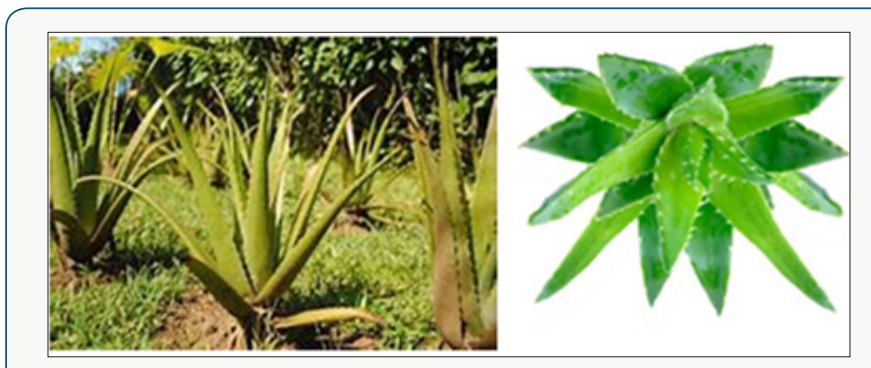

Figure 1: Aloevera leaves. 
Alovera juice extolled for its soothing qualities, it is commonly used as an ointment for burns and skin abrasions. This ancient plant may offer deeper healing abilities. Most of us are aware of the presence of aloe vera in cosmetics and skin creams; it clean skin and has anti-aging effects. People who live according to a natural health profile have long viewed the plant as a potent super food [5]. Aloe vera keep antioxidant vitamins A, C, and plus vitamin B12, folic acid, and choline. It contains eight enzymes, e.g aliases, alkaline phosphatase, amylase, bradykinase, carboxypeptidase, catalase and cellulase. Minerals such as calcium, copper, selenium, chromium, manganese, magnesium, potassium and zinc are present in aloe vera. Leaves of aloe vera provide anthraquinones. Among these are aloin and emodin, which act as analgesics, antibacterial. Fatty acids are present, including cholesterol, campesterol and beta-sitosteroll. These chemicals are responsible for reduction of copper.

\section{Materials and Methods}

\section{Material}

All the chemicals in this present study were analytical grade and pure. Aloe vera leaves were taken from nearby garden.

\section{Preparation of Plant Leaf Extract}

$30 \mathrm{~g}$ of the leaf of aloe vera plant were taken and thoroughly washed with distilled water. After washing leaves were dried and ground in morter. Boiling was next step in the preparation of extract in $100 \mathrm{ml}$ of deionized water for ten minutes at 100 degrees. After filtration solution was kept at low temperature.

\section{Green Synthesis of Cuo NPs}

A copper nitrate solution of fifty milliliters was added to $5 \mathrm{ml}$ aloe vera extract. The solution was stirred on a magnetic stirrer at 120 degrees. The color change was observed from blue to red. The resultant solution was centrifuged for ten mints at speed of 50,000rpm. After discarding supernatant copper oxide nanoparticles were dried in a watch glass. Black colored particles were collected for characterization (Figure 2).

\section{Characterization of Copper Oxide Nanoparticles}

Structural, chemical composition, size and shape of copper oxide nanoparticles were analyzed by SEM(), XRD(), EDX, TEM and UV spectrophotometer(DB-20).

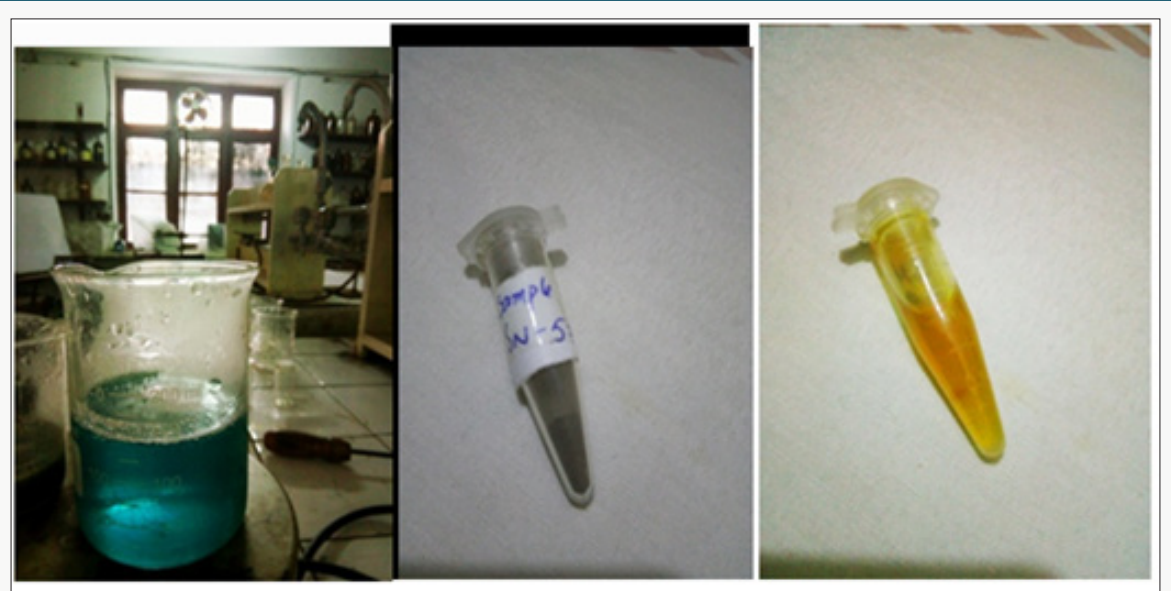

Figure 2: Copper sulphate solution, colour change cuo nanoformation, solid nano cuo particles.

\section{Result and Discussion}

\section{Color Change Observation}

Color changes indicate the formation of nanoparticles of copper oxide. The blue color solution was turned into brick red indicated for the formation of copper nanoparticles synthesis.

\section{X-Rays Diffraction Studies}

Copper oxide nanoparticles were examined by X-ray diffractometer. Copper oxide powder was put in cubes of XRD for calculation of intensity. The resultant pattern of synthesized copper oxide nanoparticles was analyzed. The peaks at $2 \theta$ intensity 32.4 , 35.6, 38.8, 48.9, 53.3, 58.2, 61, 66n and have 110, 200, 202, 020, 202, 113 and 022 patterns compare to JCPDS card. Crystal size calculated by sherrer equation found $45 \mathrm{~nm}$. Monoclinic structure was confirmed (Figure 3).

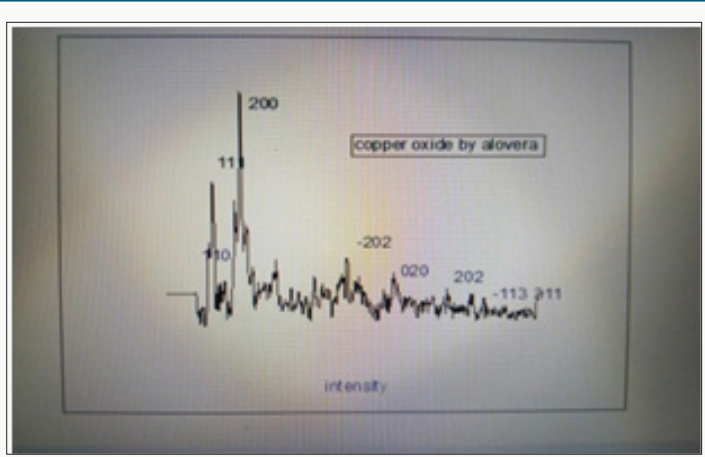

Figure 3: Xrd pattern of cu oxide nanoparticle prepare by Aloevera.

\section{Scanning Electron Microscope (SEM)}

The average particle size of copper nanoparticle was analyzed by SEM model. The copper oxide nanoparticle SEM micrograph was 
calculated about nm .It was observed that particles were smooth with a spherical shape.EDX result showed strong copper signal with other elements too like Carbon, Phosphorus (Figure 4).

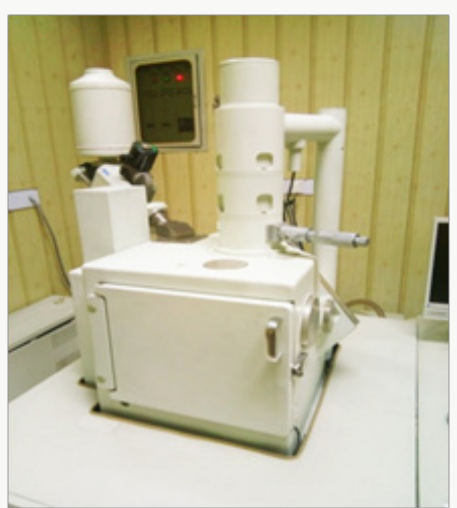

Figure 4 .
EDX Image: EDX image of aloe vera leaves stabilized copper nanoparticles shows about the composition of nanoparticles. Image showed the presence of $38 \%$ copper and \%oxygen.

FTIR Analysis: In this study, FTIR spectrum was analyzed to confirm copper nanoparticles. Peaks were observed at $1100 \mathrm{~cm}-1$ which indicates formation of copper oxide NPs. Peak was observed in range of $400-4000 \mathrm{~cm}-1$. The peaks at 3450,1600 and 2250 indicate presence of alcohol, alkene and carbon bond.

UV Visible Spectra: Peaks of UV spectrometer at a range of 200-600nm confirm the formation of copper oxide nanoparticles. Methods as it are easily available starting materials, not costly and the procedure is easy to handle in the laboratory, no toxic reagent is used and pollution free. Nanoparticles synthesized can be applicable in the different field of biochemistry, pharma, and industry (Figures 5-7).
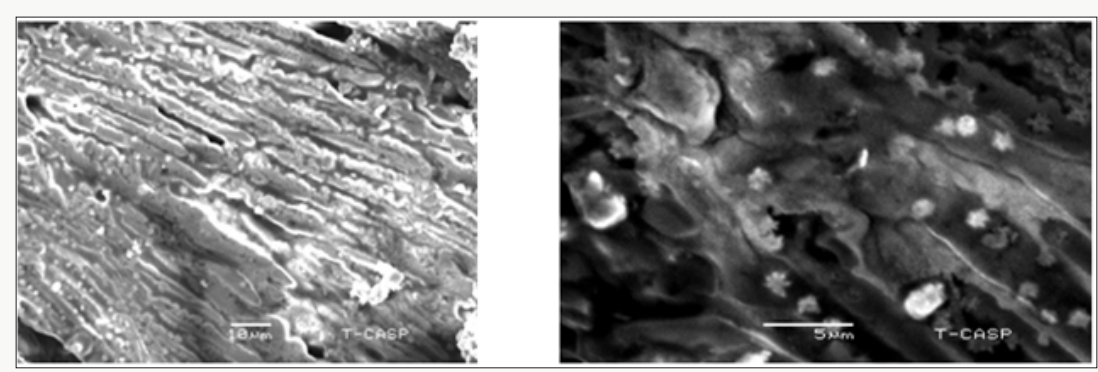

Figure 5: Sem micrograph of copper oxide nanoparticles.

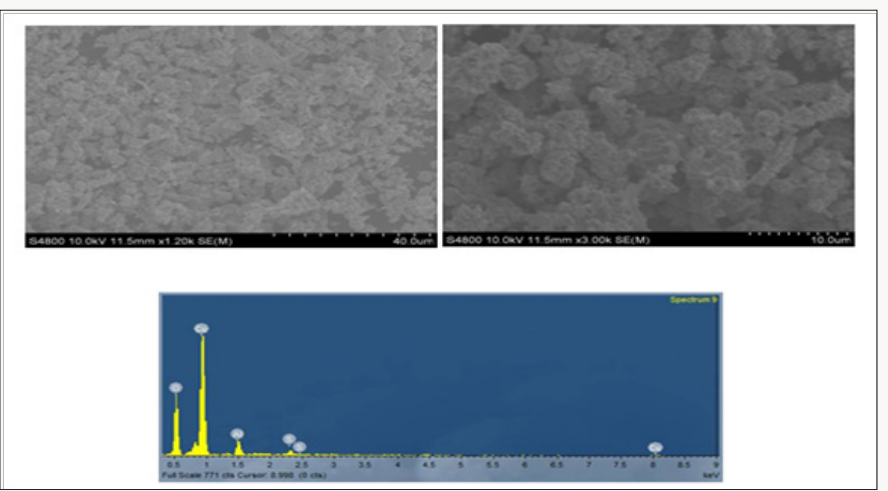

Figure 6: EDX of copper nanoparticles.
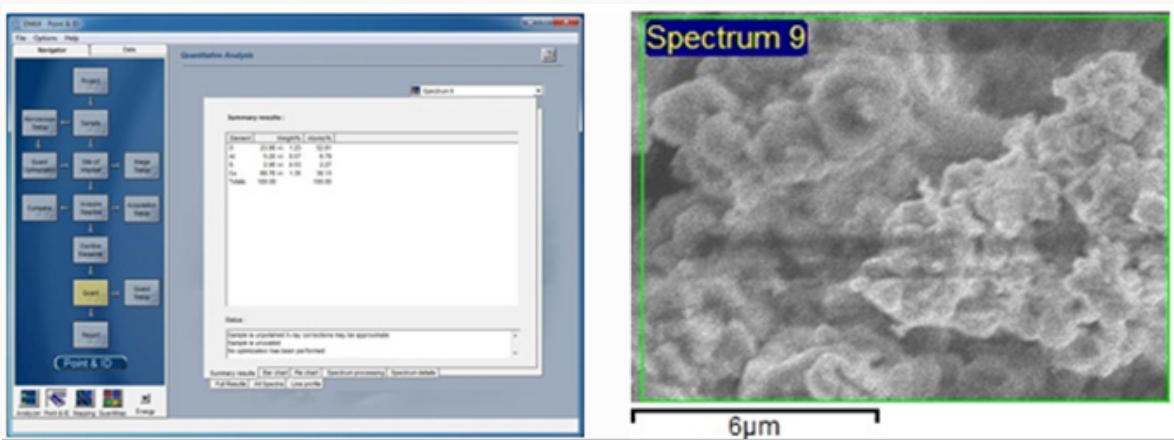

Figure 7. 


\section{Conclusion}

In conclusion, here we report an eco-friendly preparation of $\mathrm{Cu}$ nanoparticles by leaf extract of aloe vera plant. This method has an advantage over other reported methods as it is easily available starting materials, not costly and the procedure is easy to handle in the laboratory, no toxic reagent is used and pollution free. Nanoparticles synthesized can be applicable in the different field of biochemistry, pharma, and industry.

\section{References}

1. Nalwa HS (2000) Handbook of nanostructured materials and nanotechnology. New York Academic, USA.
2. Das SK, Dickinson C, Lafir F, Brougham DF, Marsili E (2012) Synthesis characterization and catalytic activity of gold nanoparticles biosynthesized with Rhizopus oryzae protein extract. Green Chemistry 14(5): 1322-1334.

3. Siavash I (2011) Green synthesis of metal nanoparticles using plants. Green Chemistry 13(10): 2638-2650.

4. Nadagouda MN, Castle AB, Murdock RC, Hussain SM, Varma RS (2010) In Vitro Biocompatibility of Nanoscale Zerovalent Iron Particles (NZVI) Synthesized using tea-polyphenols. Green Chem 12(1):114-122.

5. http://www.healthline.com/health/7amazing
(C) This work is licensed under Creative

To Submit Your Article Click Here:

Submit Article

DOI: $10.32474 /$ IPDOAJ.2018.01.000110

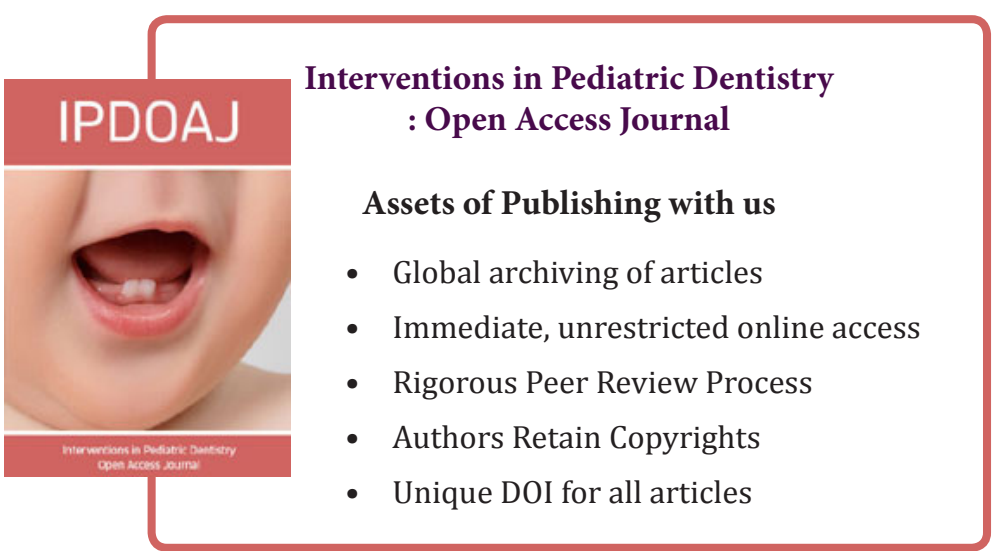

